\title{
Molecular mechanisms and clinical applications of miR-22 in regulating malignant progression in human cancer (Review)
}

\author{
JINGYU WANG ${ }^{1}$, YUAN LI $^{2}$, MEIMAN DING $^{3}$, HONGHE ZHANG $^{4}$, XIAOMING XU ${ }^{5}$ and JINLONG TANG $^{5}$ \\ ${ }^{1}$ Department of Pathology, The First Hospital of Jiaxing, Zhejiang; ${ }^{2}$ Department of Pediatrics, \\ The Affiliated Children's Hospital, Zhejiang University School of Medicine, Hangzhou; \\ ${ }^{3}$ The Criminal Investigation Detachment of Jiaxing Public Security Bureau; \\ ${ }^{4}$ Department of Pathology, Key Laboratory of Disease Proteomics of Zhejiang Province, \\ Zhejiang University School of Medicine; ${ }^{5}$ Department of Pathology, The Second Affiliated Hospital, \\ Zhejiang University School of Medicine, Hangzhou, Zhejiang, P.R. China
}

Received October 30, 2016; Accepted December 7, 2016

DOI: $10.3892 /$ ijo.2016.3811

\begin{abstract}
RNAs (microRNAs) have been validated to play fateful roles in the occurrence and development of cancers by post-transcriptionally targeting 3'-untranslated regions of the downstream gene mRNAs to repress mRNA expression. Mounting investigations forcefully document that not only does miR-22 biologically impinge on the processes of senescence, energy supply, angiogenesis, EMT (epithelial-mesenchymal transition), proliferation, migration, invasion, metastasis and apoptosis, but also it genetically or epigenetically exerts dual (inhibitory/promoting cancer) effects in various cancers via CNAs (copy number alterations), SNPs (single nucleotide polymorphisms), methylation, acetylation and even more momentously hydroxymethylation. Additionally, miR-22 expression may fluctuate with cancer progression in the body fluids of cancer patients and miR-22 could amplify its inhibitory or promoting effects through partaking in positive or negative feedback loops and interplaying with many other related miRNAs in the cascade of events, making it possible for miR-22 to be a promising and complementary or even independent cancer biomarker in some cancers and engendering profound influences on the early diagnosis, therapeutics, supervising curative effects and prognosis.
\end{abstract}

\section{Contents}

1. Introduction

2. miR-22 works as suppressor gene in tumor malignant development

Correspondence to: Dr Jinlong Tang, Department of Pathology, The Second Affiliated Hospital, Zhejiang University School of Medicine, Hangzhou, Zhejiang 310009, P.R. China

E-mail: 21018274@zju.edu.cn

Key words: miR-22, malignant progression, cancer, dual effects
3. miR-22 functions as an oncogene to promote tumor proliferation, migration and invasion

4. The functions of miR-22 participating in the feedback loops

5. miR-22 plays a critical role in EMT process in cancer

6. Molecular regulatory mechanisms of miR-22 at the genetic level in regulating tumorigenesis and malignant transformation

7. miR-22 influences cancer progression via collaborating with other miRNAs

8. miR-22 functions as a sensitizer in cancer treatments

9. miR-22 is an independent biomarker for cancer diagnosis, surveillance and prognosis

10. Conclusions and prospects

\section{Introduction}

miR-22, primitively cloned from HeLa cells, is an evolutionarily-conserved gene located in chromosome 17p13, its cDNA catalyzed by RNA polymerase II is $\sim 1.3 \mathrm{~kb}$ and promoter TSS (transcription start site) lacks TATA box (1). Recently, increasing numbers of studies have confirmed that miR-22, to a large extent, determines the destiny of many cancers, to die soon or survive, by the complicated known or unknown mechanisms through targeting and suppressing downstream transcription factors. Frequently, since miR-22 in different contexts are aberrantly expressed upregulation or downregulation in various cancers, such as prostatic cancer, esophageal squamous cell carcinoma, breast cancer, and gastric cancers, thus miR-22 shows different effects in these cancers (2-5), that is, it served not only as a tumor-suppressive miRNA, but also as an oncogenic miRNA to encumber or aggravate cancer formation and malignant transformation $(3,6)$. Besides, reports also showed that miR-22 may prominently influence cancer biological behaviors, such as proliferation, invasion and metastasis $(7,8)$, and it genetically alters expression of numerous related genes (9), which unveils the intrinsic mechanisms of miR-22 in regulating cancer formation by means of 
multi-approaches and multi-layers, indicating the central roles of miR-22 in manipulating the occurrence and development of different cancers. Since the underlying regulatory mechanisms of miR-22 are complicated and remain poorly expounded, we concentrated on the potential mechanisms and clinical applications of miR-22 in modulating cancer progression.

\section{2. miR-22 works as suppressor gene in tumor malignant development}

miR-22 inhibits tumor proliferation, invasion and metastasis by accelerating cell senescence, inhibiting energy metabolism and angiogenesis. Considering that tumor progression, including proliferation, invasion and metastasis, are intimately involved in tumor growth status and energy supply, miR-22 could interrupt these processes by mediating tumor growth status and energy supply. For instance, miR-22 induced p53 expression and concurrently targeted SIRT1, CDK6 and Sp1 to activate $\mathrm{pRb}$ signaling pathway, thereby hastening senescence, retarding cellular growth, invasion and metastasis in cervical cancer and breast cancer, which shows evident anticancer effects (8) (Fig. 1).

Glucose is one of the crucial energy sources, and angiogenesis conveys energy and nutrient for rapid cancer growth beyond the restrictions of original blood supply. Congruently, miR-22 could cut off energy metabolism by directly silencing GLUT1 (glucose transporter protein type 1), a protein unidirectionally transferring glucose into the cytoplasm to promote energy metabolism, and ACLY (ATP citrate lyase), an enzyme accelerating lipid synthesis and elevated expression in cancers, restraining cancer proliferation, migration and invasion and inducing apoptosis, which is negatively linked to TNM stage, metastasis, recurrence and survival rates of breast, prostate, osteosarcoma, lung and cervical cancers $(10,11)$. Moreover, miR-22 has low expression in colorectal cancer (CRC), and increased expression of miR-22 to silence HIF-1 $\alpha$ (hypoxia inducible factor $1 \alpha$ ) may severely repress VEGF (vascular endothelial growth factor) expression to block angiogenesis, leading to the disruption of cancer progression (12) (Fig. 1).

Considering the above, one of the feasible methods to effectively disrupt tumor formation may be by elevating miR-22 expression to hasten senescence, cut energy supplies and block angiogenesis.

miR-22 inhibits tumor proliferation, invasion and metastasis via repressing tumor cell cycle and promoting apoptosis. It is universally known that numerous cancer cells continuously enter proliferation and division through G0/G1 checkpoint along with reducing apoptosis, eventually causing rapid cancer growth and enlargement in size. Recently, extensive evidence has demonstrated that miR-22 could repress tumor malignant process by inhibition of the cell cycle. For example, miR-22 may post-transcriptionally target cyclin $\mathrm{A} 2$ and CDKN1A (cyclin-dependent kinase inhibitor 1A) to arrest the cell cycle in G0/G1 phage in CRC and liver cancer, respectively $(13,14)$. Besides, augmenting expression of miR-22 in ER (estrogen receptor) $\alpha$-positive endometrioid adenocarcinoma where miR-22 expression is usually low could downregulate ER $\alpha$ expression to further decrease the expression of cyclin D1 and member matrix metalloproteinase 2/9 (15). Furthermore, carcinogen TPA (12- $O$-tetradecanoylphorbol-13-acetate)induced miR-22 may inversely regulate PKC/ERK pathway via dramatically downregulating Max (a transcription factor binding to and activate c-Myc) expression, thus resulting in G0/G1 arrest in lung, breast and prostate cancer cells (16). These findings confirmed that the ultimate effects of miR-22 by cell cycle arrest in different ways may lead to the attenuation of cancer growth and invasion and the disruption of tumor malignant progression, indicating that miR-22 in response to different carcinogens may serve as a tumor suppressor miRNA to mitigate or block cancer occurrence and development.

Several emerging studies have validated that miR-22 could influence tumor proliferation by regulating hormone-related signaling pathway. For instance, the proliferation and migration of CRC cells and ER $\alpha$-positive breast cancer cells may be attenuated by $1,25(\mathrm{OH})_{2} \mathrm{D}_{3}$ (the in vivo metabolite of vitamin D3)-induced miR-22 and by ectopic introduction of miR-22, respectively $(17,18)$, insinuating that the regulation of miR-22 participating in hormone signal transduction pathway should not be ignored as critical underlying mechanism for tumorigenesis and progression.

Furthermore, miR-22 may also contribute to the cessation of cancer aggression through post-transcriptionally regulating downstream molecules with respect to cellular migration and adhesion, a case in point is that miR-22 in gastric cancer may separately silence CD151, a molecule promoting cellular migration, and MTDH (metadherin), a molecule involved in cellular adhesion, to effectively interfere with cancer cellular proliferation and metastatic dissemination $(19,20)$.

Additionally to the above, miR-22 has been reported to tightly repress cellular immune escape and proliferation and trigger apoptosis by targeting a variety of downstream molecules, such as Galectin-9, NET1 (neuro-epithelial transforming gene 1) and PAPST1, in liver cancer, chronic myeloid leukemia (CML) and medulloblastoma, respectively (21-23). In addition, not only could curcumin-induced miR-22 posttranscriptionally degrade oncogene Erbb3 in retinoblastoma, but also ectopic elevated miR-22 expression could directly target Erbb3 or EVI-1, subsequently causing the repression of PI3K/Akt cascade, the end result is inhibition of cellular proliferation, migration, invasion and metastasis in lung cancer and breast cancer, respectively (24-26). Moreover, miR-22-mediated silencing of ESR1 and TIAM1 may directly repress cancer cellular migration and invasion without exerting effects on cellular viability and apoptosis in metastatic ovarian cancer (27).

To summarize, miR-22 shows considerable antitumor effects via various and synthetic rather than only a single mechanism to intervene in multi-step processes of tumorigenesis (Fig. 1).

\section{3. miR-22 functions as an oncogene to promote tumor proliferation, migration and invasion}

Conversely, in some cancers, miR-22 may serve as a crucial driver to promote rather than inhibit cancer aggressiveness. An example is that IL-1 $\alpha$-stimulated miR-22 may initiate 


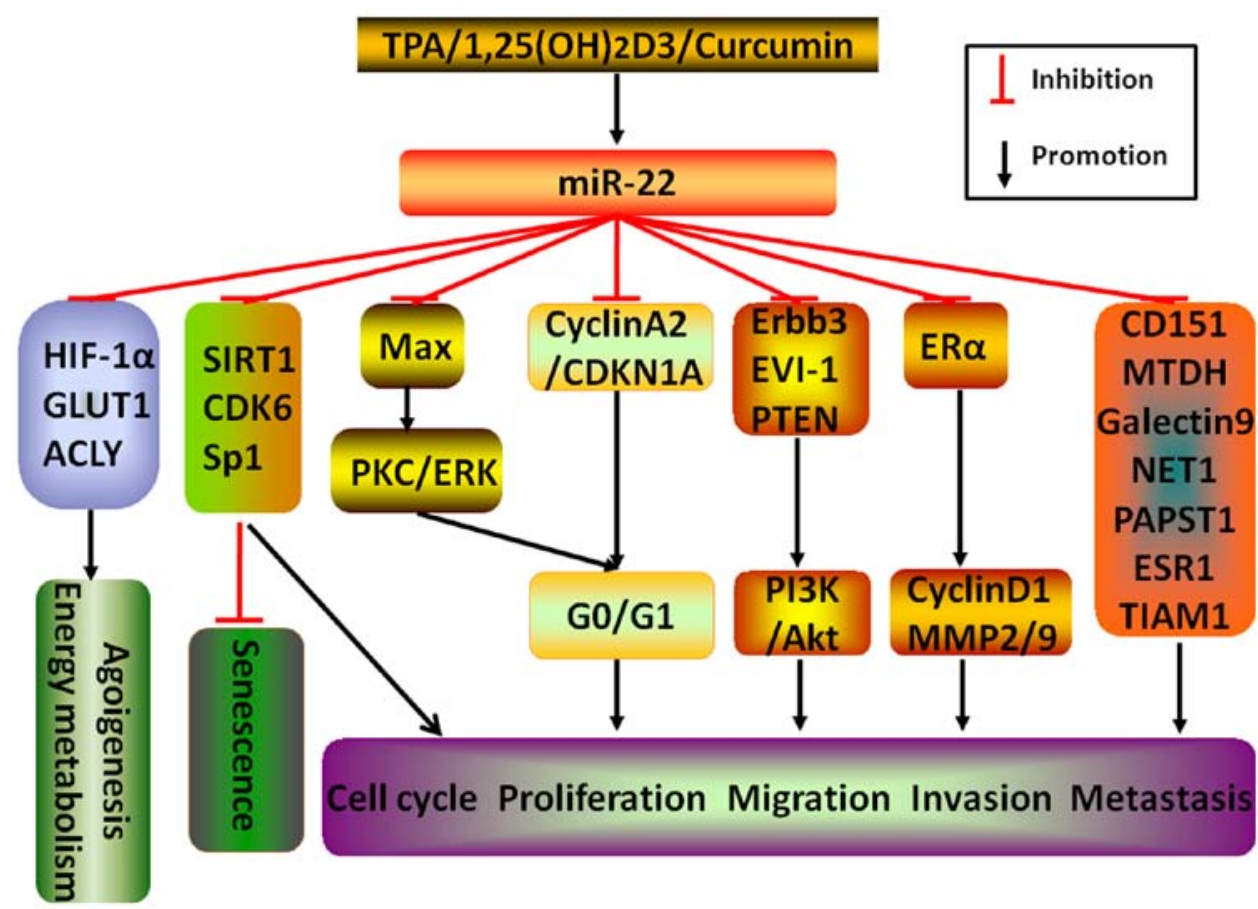

Figure 1. miR-22 inhibits tumor malignant progressions. Different stimulators, including TPA, $1,25(\mathrm{OH})_{2} \mathrm{D}_{3}$ and curcumin, induce miR-22 expression. Through targeting various downstream related molecules, such as HIF-1 $\alpha$, GLUT1, ACLY, SIRT1, CDK6, Sp1, CD151, MTDH, Galectin-9, NET1, PAPST1, ESR1, TIAM1, Max, Cyclin A2/CDKN1A, Erbb3, EVI-1, PTEN and ER $\alpha$, miR-22 is capable of directly or indirectly abrogate the process of tumor malignancy, including acceleration of senescence and the abruption of angiogenesis, energy metabolism, cell cycle, proliferation, migration, invasion and metastasis.

HBV-related liver cancer by suppressing ER $\alpha$ (28), and another example is that by directly targeting PTEN (phosphatase and tensin homolog, a gene usually regarded as tumor suppressor factor), miR-22 in clear cell renal cell carcinoma where its expression is frequently downregulated has been shown to abolish cancer proliferation, migration and invasion but in prostate cancer where its expression is high to stimulate $(7,29)$, the most important reason may be explained by a further well-performed study in CLL (chronic lymphocytic leukemia) that miR-22-targeted PTEN silencing may spontaneously activate PI3K/AKT pathway to the downregulated expression of p27 (-Kip1) and upregulated expression of Survivin, CCND2 (Cyclin D2) and MAPK1 (mitogen-activated protein kinase 1), thereby resulting in the end telling effects of tumor formation (30) (Fig. 2).

Collectively, adequate access to tumorigenesis of miR-22 in some tumors is achieved by modulating tumor suppressor molecules to initiate oncogene-related signal cascade events, therefore, in these tumors, repressing miR-22 expression to persistently inactivate downstream interactive oncogenic molecules may be an effective means of preventing tumor proliferation, migration and invasion.

\section{The functions of miR-22 participating in the feedback loops}

Intriguingly, in the form of positive or negative feedback loops, numerous miRNAs, such as miR-200, miR-203 and miR-183/96/182, play pivotal roles in intimately inhibiting or promoting cancer occurrence and development (31-34), and so does miR-22. For instance, in cervical cancer and breast

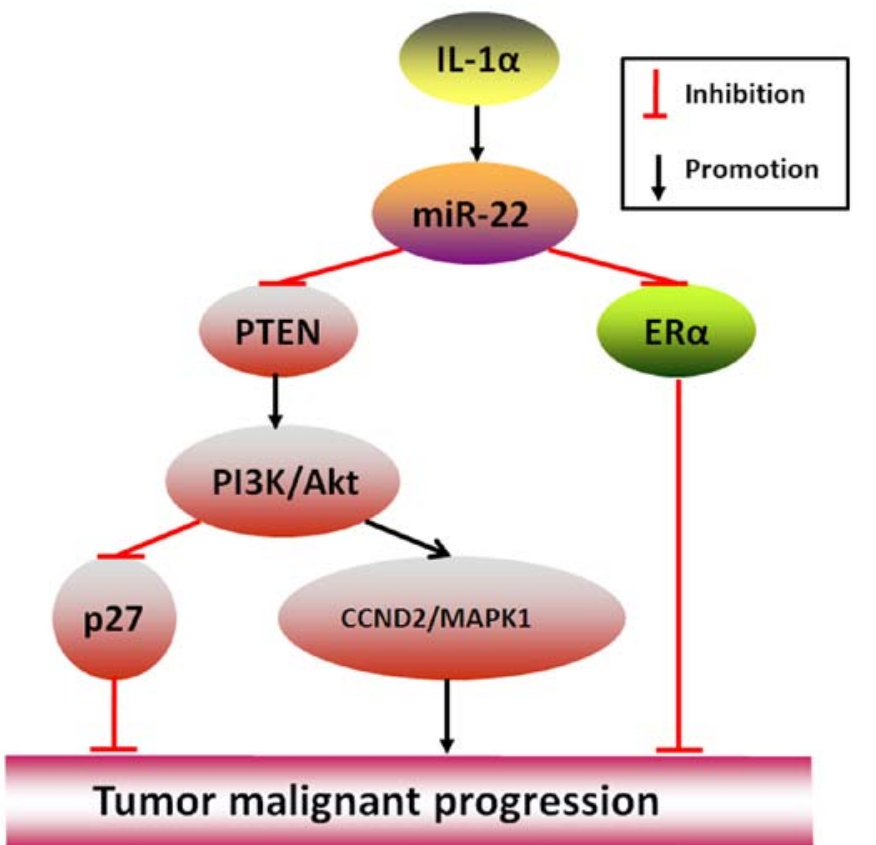

Figure 2. miR-22 services as onco-miRNA to promote tumor malignant transformation. IL-1 $\alpha$-induced miR-22 may initiate cancer by silencing $E R \alpha$, and it could as well activate PI3K/AKT pathway to downregulate p27 expression and upregulate CCND2 and MAPK1 expression by repressing PTEN, thus giving rise to tumor aggressiveness.

cancer, miR-22 has been revealed to act as an onco-miRNA to directly target PTEN and subsequently initiate PI3K/AKT/ FoxO1 pathway. Nevertheless, the activated AKT unexpect- 
edly induces miR-22 expression, eventually forming a positive feedback loop and continuously simulate PI3K/AKT/FoxO1 cascade to promote tumor malignant transformation (6). In addition, binding of MYCBP (c-Myc-binding protein) to inactive c-Myc which is capable of repressing miR-22 expression, miR-22 forms a strongly positive feedback loop in favor of the inhibition of breast cancer growth (35). Moreover, additional experimental results have hinted that besides straightly degrading transcription factor SP1 to retard the migration and invasion of gastric cancer (36), miR-22 may as well concurrently target and inactive CD147 (an inducible factor of extracellular matrix metalloproteinase) and SP1, but both SP1 and c-Myc are capable of binding to the promoter region of CD147 and subsequently enhancing CD147 expression, and simultaneously of miR-22 and inhibiting miR-22 expression, which constitutively promotes expression of SP1 and c-Myc to upregulate CD147 expression, finally facilitating the proliferation, migration, invasion and metastasis of breast cancer. Oppositely, the reverse could be observed as miR-22 expression was increased (37) (Fig. 3).

Taking the data collectively, miR-22 undertakes the kernel role in controlling the proliferation, migration, invasion and metastasis of different cancers by closely intertwining with multiple tumor suppressor genes or oncogenes of upstream or downstream molecules to form positive or negative feedback loops. However, since miR-22 has dual (inhibitory or promoting) functions in different cancers, especially breast cancer, in different experiments, it is therefore necessary to further elucidate the underlying mechanisms of miR-22 in regulating feedback loops, correspondingly augmenting or reducing miR-22 expression in different cancers, particularly breast cancer, will be maximally instrumental for amplifying its anticancer effects or restricting its accelerated effects.

\section{5. miR-22 plays a critical role in EMT process in cancer}

Surprisingly, increasing findings have documented a fascinating and usually ignored mechanism of miR-22 with reference to the regulation of EMT, a process expediting cancer invasion and metastasis and shifting cells from an epithelial status to a mesenchymal status accompanied by morphological loss of cohesiveness and an increased motility and genetically the downregulated expression of epithelial adhesive molecules, including E-cadherin and ZO-1, and upregulated expression of mesenchymal molecules, including Zeb1/2, snail1/2, Vimentin, Twist (38). As examples, miR-22 is capable of promoting cancer proliferation, migration and incursion by potently silencing acetylase TIP60, a gene inhibiting cancer formation, which is significantly correlated with the worse prognosis of patients with breast cancer (39). Notwithstanding, miR-22 has been reported to markedly attenuate EMT process and cancer distant metastasis by directly targeting TIAM1 (T-cell lymphoma invasion and metastasis 1), a gene augmenting expression of MMP2/9 (member matrix metalloproteinase 2/9) to exacerbate cancer invasion and metastasis, MMP14 (member matrix metalloproteinase 14), Snail and SIRT1 in CRC, gastric cancer and RCC, respectively (40-42) (Fig. 4). Moreover, the downexpression of miR-22 in folate deficiency HCC cells may lead to the upexpression of its target gene Zeb2, which may be related to the initiation of EMT process (43).
Given miR-22 could directly target either EMT-associated tumor suppressors or oncogenes to induce or debilitate EMT progression and metastasis, sustained and targeted upregulation or downregulation of miR-22 in various cancer types may forcefully cease the EMT process and distant metastasis, thereby displaying optimal therapeutic effects on patients with malignant cancers.

\section{Molecular regulatory mechanisms of miR-22 at the genetic level in regulating tumorigenesis and malignant transformation}

Strikingly, accumulating findings have revealed the link between epigenetic abnormalities and miR-22 (Fig. 5). The gene CNAs and SNPs may significantly impact on the modulation of miR-22 to different cancers. As an example, 2/21 cases of acute lymphoblastic leukemia patients have miR-22 copy number deletions at $17 \mathrm{p} 13.3$ (44), and in cervical cancer, the failed binding of miR-22 to rs11064 variant GG allelic genotype of TNFAIP8 (tumor necrosis factor- $\alpha$-induced protein 8 , a target of miR-22) will distinctively result in the overexpression of TNFAIP8 and subsequently against apoptosis and facilitate unimpeded tumor formation, which is highly pertinent to platinum resistance (45), evincing that the persons suffering copy number deletions of miR-22 gene or SNP alterations of miR-22 target genes may be much more vulnerable to malignant tumors than the normal ones.

Growing number of mechanistic experiments documented that miR-22 may control cancer proliferation and growth through epigenetically modulating histone acetylation, DNA methylation and gene repair. In hepatocellular carcinoma and Ewing sarcoma, externally induced expression of miR-22 has been shown to repress cellular proliferation and tumorigenesis via post-transcriptionally silencing histone deacetylase HDAC4 (histone deacetylase 4) and KDM3A (lysine (K)-specific demethylase $3 A$ ), respectively $(46,47)$. Generally, promoter hypermethylation implies the silence of gene expressions, inversely, promoter demethylation hints at promotion of gene expressions. In androgen receptorpositive cancer cells of prostate cancer, miR-22 and miR-29 promoters are frequently hypermethylated which makes their expression low. Whereas, cancer cells will obviously undergo restrained migration and stimulated apoptosis by upregulated miR-22 and miR-29 to separately target LAMC1 (laminin $\gamma 1$, a gene promoting cell migration) and MCL1 (myeloid cell leukemia 1, a gene against apoptosis) (9). Additionally, AKT-induced miR-22 in CRC HCT116 has been reported to directly target MDC1 (mediator of DNA damage checkpoint 1), giving rise to the aberrant repair of damaged DNA and instability of genes, thus, causing probable susceptibility to aging and cancer (48).

Extremely absorbing, miR-22 has been shown to participate in tumorigenesis via regulating 5-hmC (5-hydroxymethylcytosine), usually called the six base and originating from $5-\mathrm{mC}$ (5-methylcytosine) catalyzed by TET (ten-eleven translocation) enzymes TET1, TET2 and TET3. 5-hmC of transcription factor binding sites commonly initiates gene expression, and the downexpression of TET often fails to activate gene transcription owing to the low level of 5-hmC $(49,50)$. For instance, analysis of patients with refractory cytopenia of childhood revealed 


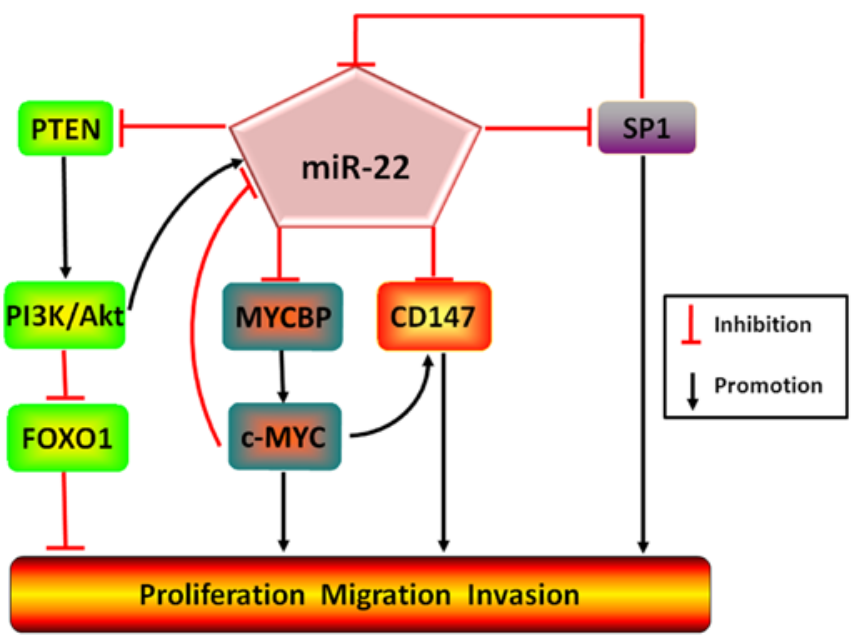

Figure 3. Functions of miR-22 participating in the reciprocal feedback loops. miR-22 could be regarded as an oncogene due to the positive feedback loop between miR-22 and PI3K/Akt to promote tumor malignant transformation. Inversely, it could be viewed as a tumor suppressor gene owing to the negative feedback loop between miR-22 and SP1 and c-MYC, which eventually inhibits CD147 expression available to the cessation of malignant transformation. that the high expression of miR-22 has a closely converse relationship with the low expression of TET and 5-hmC (51). Furthermore, recent investigations have confirmed in miR-22 transgenic mice that by directly targeting TET 2 , not only is miR-22 against methylation of tumor suppressor miR-200 promoter, causing the downregulation of miR-200 and the initiation of EMT process and distant metastasis for breast cancer stem cells, which is closely correlated with the poor prognosis of patients (52), but also it contributes to the low levels of other 5-hmC of downstream genes, leading to selfrenewal and malignant transformation of blood stem cells in these mice, which eventually undergo MDS and malignant blood diseases. Instead, miR-22 depression may lead to the inhibitory proliferation of leukemia cells in mice and human (53). Nonetheless, the latest investigation hinted that in AML (acute myeloid leukemia) cells, the expression of TET2 has a positive rather than negative relation with the expression of miR-22, but upregulated TET1 has a negative association with the expression of miR-22. Although TET1 is conducive to the hypomethylation of miR-22 promoter, it, strangely, does not induce miR-22 expression, the primary reason may be that both copy number deletions of miR-22 gene and

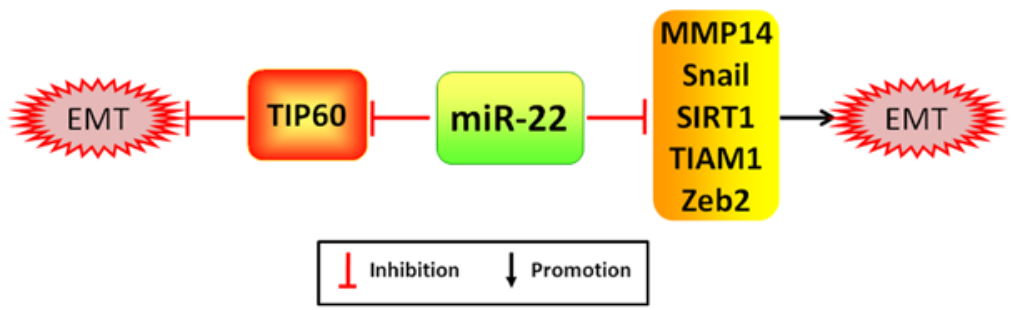

Figure 4. Critical role of miR-22 in regulating EMT process. miR-22 may either facilitate or retard EMT process by directly silencing TIP60, TIAM1, MMP14, Snail, SIRT1 and Zeb2, respectively.

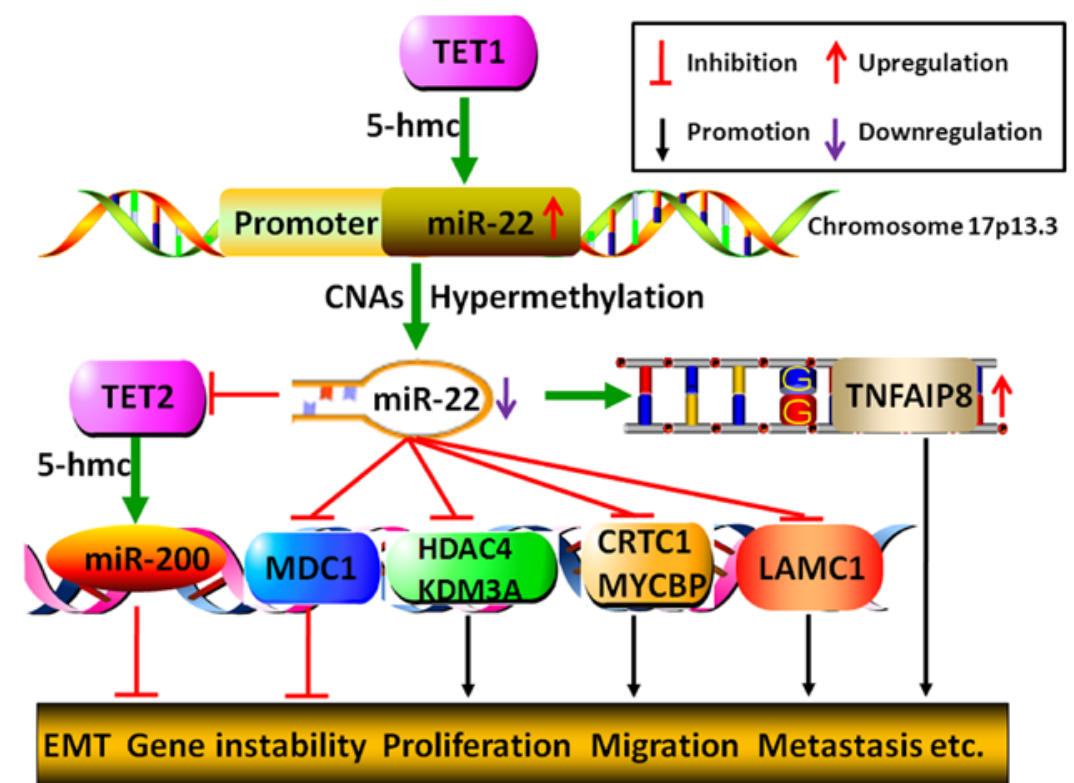

Figure 5. Genetical or epigenetical mechanisms by which miR-22 regulates tumor malignant progression. miR-22 gene 5-hmc catalyzed by TET1 may lead to its increased expression, whereas, either CNAs or hypermethylation of miR-22 promoter may bring about its decreased expression. Moreover, miR-22 could restrain miR-200 gene 5-hmc to initiate EMT process by directly degrading TET2. Additionally, miR-22 may fail to inhibit cancer process due to its target gene TNFAIP8 SNPs-GG allelic genotype, which results in the upregulation of TNFAIP8. Furthermore, by modulating histone acetylation, DNA methylation and gene repair-related molecules, MDC, HDAC4, KDM3A, CRTC1, MYCBP and LAMC1, miR-22 could either inhibit or promote tumor malignant transformation, including gene instability, proliferation, migration, invasion and metastasis. 


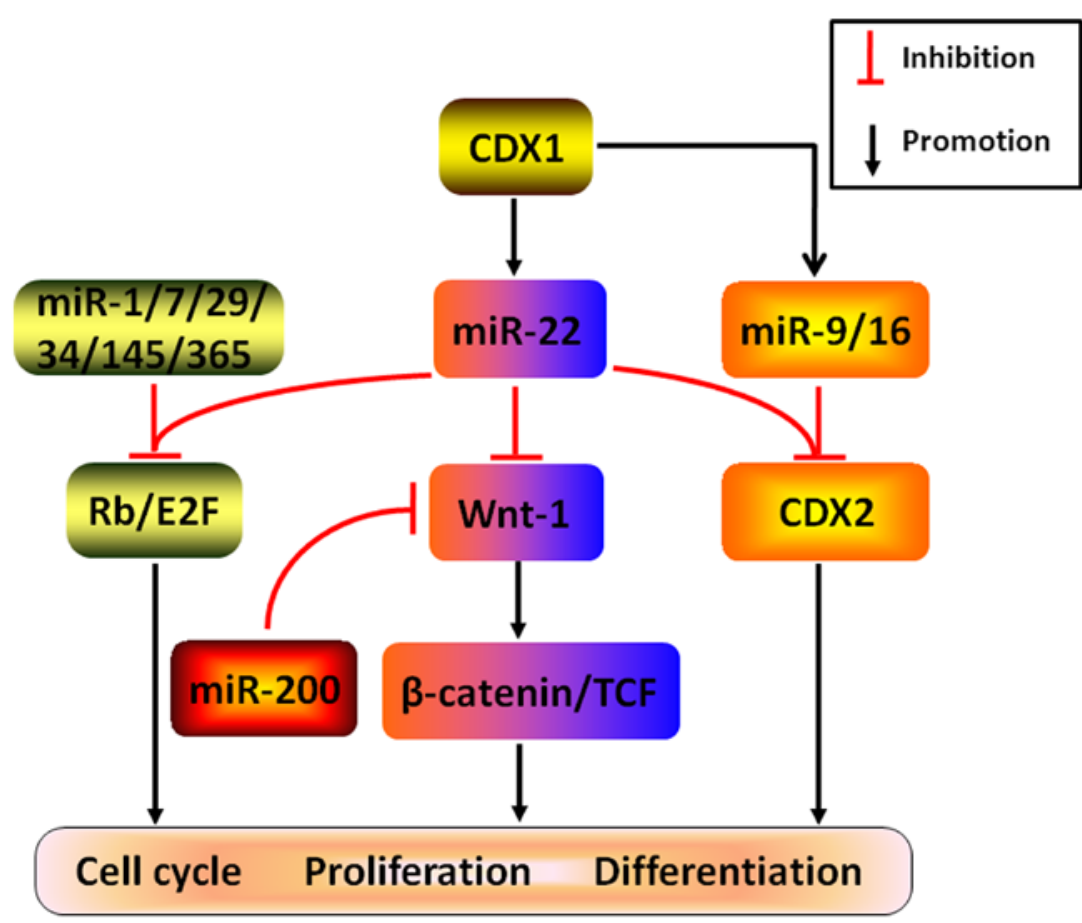

Figure 6. Collaborating effects of miR-22 with other miRNAs. Cooperating with many other anticancer miRNAs, such as miR-1/7/29/34/145/365, miR-200 and miR-9/16, to silence the same target genes Rb/E2F, Wnt-1 and CDX2, respectively, CDX1-induced miR-22 may significantly inhibit cell cycle, proliferation and differentiation.

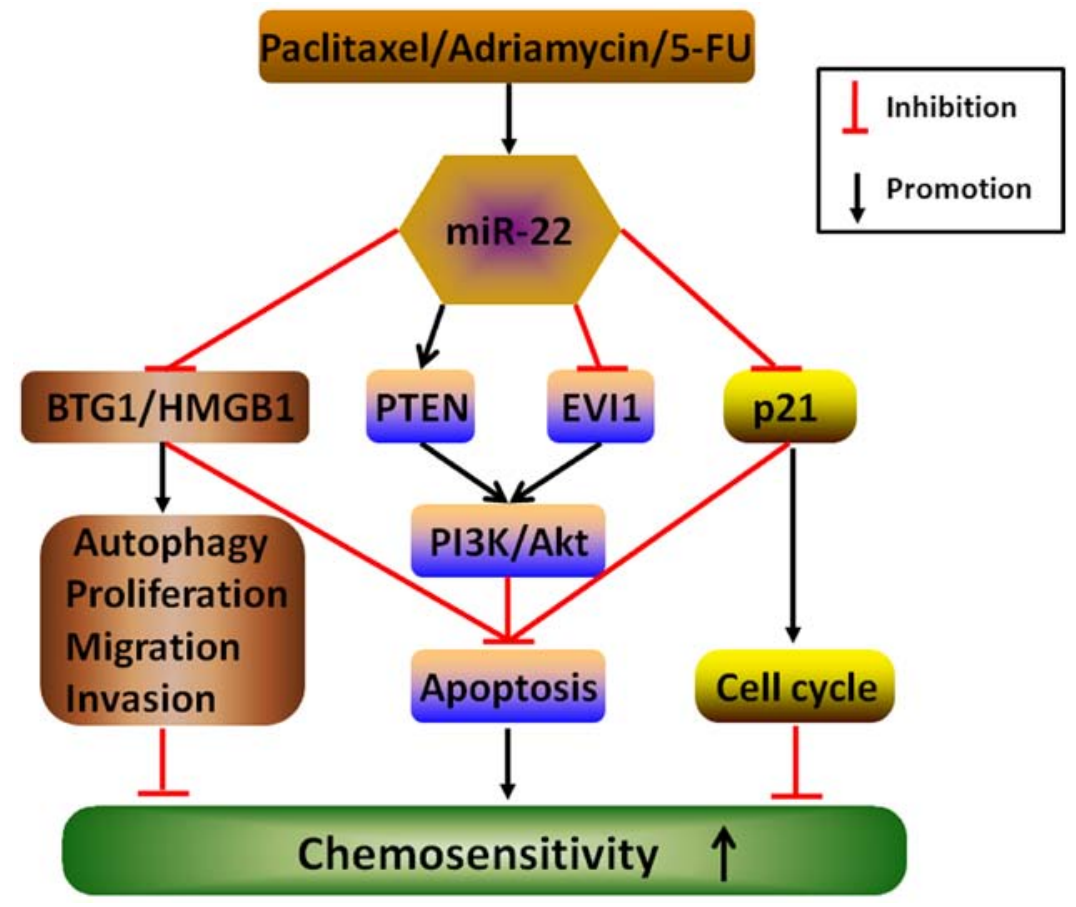

Figure 7. Underlying mechanisms by which miR-22 enhances chemosensitivity to therapeutic drugs in cancers. The elevated expression of miR-22 stimulated by anticancer drugs, including paclitaxel, adriamycin and 5-FU, may directly target and activate or inactivate multiple downstream molecules, such as BTG1, HMGB1, PTEN, EVI1 and p21, to counteract autophagy, proliferation, migration and invasion and cell cycle and induce apoptosis, thereby enhancing chemosensitivity in different cancers.

binding of upstream cofactors GFI1/EZH2/SIN3A and TET1, especially TET1, to the region of miR-22 promoter result in the inhibition of miR-22 expression. However, restoration of miR-22 may markedly abate the CREB and MYC pathways by directly targeting CRTC1 (CREB-regulated transcription coactivator 1) and MYCBP, bringing about the inhibition of tumor formation and malignant transformation (54). Briefly, these data suggest that miR-22 may directly be controlled 
Table I. Clinical applications of miR-22 in tumor diagnosis, surveillance and prognosis.

\begin{tabular}{llcccc}
\hline Tumor & Body fluids/tissues & miR-22 levels & Diagnosis & Treatment response/prognosis & Refs. \\
\hline ICC & Serum & $\downarrow$ & $\sqrt{ }$ & $\mathrm{N}$ & $(63)$ \\
Hepatocellular carcinoma & Serum & $\downarrow$ & $\sqrt{ }$ & Poor & $(46,64)$ \\
Lung adenocarcinoma & Pleural effusion & $\downarrow$ & $\sqrt{ }$ & $\mathrm{N}$ & $(65)$ \\
ESCC & Serum & $\uparrow$ & $\sqrt{ }$ & $\mathrm{N}$ & $(66)$ \\
Pancreatic cancer & Serum & $\uparrow$ & $\sqrt{ }$ & $\mathrm{N}$ & $(67)$ \\
Prostatic cancer & Serum & $\uparrow$ & $\sqrt{ }$ & N & $(2)$ \\
NSCLC & Serum & $\uparrow$ & $\mathrm{N}$ & Poor & $(68)$ \\
Gastric cancer & Cancer tissue & $\downarrow$ & $\mathrm{N}$ & Poor & $(69)$ \\
CRC & Cancer tissue & $\downarrow$ & $\mathrm{N}$ & Poor & $(70)$ \\
\hline
\end{tabular}

ICC, intrahepatic cholangiocarcinoma; ESCC, esophageal squamous cell carcinoma; NSCLC, non-small cell lung cancer; CRC, colorectal cancer; $\downarrow$, low level; $\uparrow$, high level; $\sqrt{ }$, valuable; $N$, no report.

by hydroxymethylation, but it does not necessarily mean that the hypomethylation of miR-22 promoter leads to the upregulation of miR-22, to some extent, many other factors, including copy number deletions of miR-22 gene and the direct inhibition of miR-22 promoter by hydroxymethylationrelated genes, are prominently correlated with suppressing miR-22 expression, miR-22 may turn off the expression of downstream suppressor genes with respect to the hydroxymethylation-related pathways, thereby playing an oncogenic role in the process of tumor initiation and malignant transformation.

Taken together, at the genetic level, on the one hand, copy number deletions or hypermethylation of miR-22 gene may result in its dysfunction to facilitate tumor uncontrolled malignant progression, indicating that miR-22 likely serves as a tumor suppressor to design epigenetic drugs in some cancers. On the other hand, miR-22 could bring about genomic instability to promote tumor malignant transformation via manipulating epigenetic modification to turn off the expression of tumor suppressors, suggesting that miR-22 probably functions as an internal engine for some cancers. As so many are underlying mechanisms involved in miR-22 participating in cancer formation that it is necessary to genetically explore and unveil the intricate mechanisms of miR-22 for epigenetic therapy in different cancers.

\section{7. miR-22 influences cancer progression via collaborating with other miRNAs}

Tantalizingly, increasing evidence has recently corroborated that the combined effects of miR-22 complexing with many other miRNAs may play a crucial role in controlling cancer differentiation, proliferation, migration and invasion in some cancers (Fig. 6). Considering several examples, experimental validation demonstrated that both CDX1-induced miR-22, miR-9 and miR-16 may directly target CDX2 to abrogate cell differentiation in CRC (55). In addition, cooperating with several other miRNAs, such as miR-1, Let-7, miR-29, miR-34, miR-145, and miR-365, miR-22 was found to attenuate $\mathrm{Rb} / \mathrm{E} 2 \mathrm{~F}$ signaling pathway by post-transcriptionally silencing $\mathrm{Rb}$ to remarkably arrest cell cycle and DNA replication, ultimately resulting in the restriction of cancer growth (56). Likewise, by coordinating with tumor suppressor gene miR-200 and directly targeting Wnt-1, an oncogene positively modifying Wnt/ $\beta$-catenin pathway, miR-22 is capable of repressing the expression of $\beta$-catenin and TCF to potently restrain cancer colony-forming, which may be responsible for the anticancer effects in gastric cancer (57).

Overall, these findings indicate that it is perhaps a feasible way to force expression of the miR-22 combining with many other miRNAs regarding the repression of carcinoma-related pathways to strengthen anticancer effects in some cancers, opening a new window for unearthing the intrinsic underlying mechanisms of miR-22 in modulating the cancer progression.

\section{8. miR-22 functions as a sensitizer in cancer treatments}

Clinically, it is very common that the same chemotherapeutic drugs show extraordinarily different therapeutic effects on different cancers even on the same cancers owing to chemoresistance which is one of the most imperative reasons for the failure of treatment, the toughest challenges and seemingly insurmountable obstacles. Utterly inspiring is the good news that miR-22 in several cancers, to some extent, displays the capability of increasing chemosensitivity to different anticarcinogens by directly targeting and activating or inactivating various downstream genes (Fig. 7). Findings from recent experiments have confirmed that $\mathrm{p} 53$-wild rather than p53-mutant type CRC cells display chemoresensitivity to paclitaxel partly in that enhanced miR-22 binding to and activating PTEN can counteract a cascade of PI3K/Akt events and stimulate apoptosis, leading to resensitization to paclitaxel (58). Further research revealed that via binding to the 5-upstream regions and intron regions of C17orf91, a gene in which miR-22 locates, adriamycin-triggered p53 in p53-wild-type CRC cells is capable of augmenting miR-22 expression to target downstream molecule p21 to subsequently cease the cell cycle and induce cell apoptosis, ultimately resulting in intensifying the chemosensitivity to 
adriamycin (59). In addition, there were several interesting and important mechanisms, including autophagy pathway and apoptosis pathway, with respect to miR-22 in enhancing sensitivity to several chemotherapeutics. Through separately targeting BTG1 (B-cell translocation gene 1) and HMGB1 (high-mobility group box 1), miR-22 could inhibit autophagy and enhance apoptosis against proliferation, migration and invasion, eventually contributing to the reverse chemoresistance to 5-FU, cisplatin and doxorubicin in CRC and osteosarcoma $(60,61)$.

Moreover, miR-22 may profoundly enhance chemosensitivity to anticancer drugs by intimately working with many other miRNAs. In clear cell ovarian cancer, PI3K/Akt/mTOR pathway was predominately repressed due to the combined effects through directly targeting both FGFR3 (fibroblast growth factor receptor 3) and mTOR by miR-100 and simultaneously targeting EVI1 by miR-22, allowing for the subsequent inhibition of proliferation, growth and survival, which, in the end, increases chemosensitivity to anticancer drug everolimus (62).

Taken together, these data hint that miR-22 may notably enhance chemosensitivity or reverse chemoresistance to corresponding cancer drugs by a variety of underlying mechanisms, and that multiple anticancer drugs with predominately therapeutic effects may further maximize their advantages or minimize disadvantages via augmenting miR-22 expression to exert the inhibition of cancer proliferation, migration and invasion. Therefore, to obtain the optimal treatment effects for miR-22 as an adjuvant therapeutic intervention in the near future, illuminating the potential mechanisms of miR-22 in regulating chemosensitivity or chemoresistance will be beneficial for patients with various cancers in precision medicine.

\section{9. miR-22 is an independent biomarker for cancer diagnosis, surveillance and prognosis}

Early diagnosis is one of the primary challenges of cancers and would be beneficial for effectively prolonging the survival time and improving the patient life quality. As miR-22 expression is different in different cancers and plays a central role in cancer cell proliferation, migration, invasion and metastasis, it is reflected in cancer diagnosis, monitoring, and prognosis. For instance, the low expression of miR-22 in the serum of patients with ICC (intrahepatic cholangiocarcinoma) and hepatocellular cancer with hepatitis $C$ virus and the malignant pleural effusion of patients with lung adenocarcinoma may well be promising as an independent early diagnostic biomarker for these cancers (63-65). On the contrary, the sustained high expression of miR-22 in the serum of patients with esophageal squamous cell carcinoma, pancreatic cancer and metastatic prostatic cancer may well be a reliable serum biomarker for cancer diagnosis, along with the desirable diagnosis of sensitivity and specificity $(2,66,67)$ (Table I).

Additionally, the alteration of miR-22 expression in body fluids may, to a certain extent, directly mirror the therapeutic effects. The elevated expression of miR-22 in the serum of NSCLC (non-small cell lung cancer) is conspicuously associated with the cancer aggression and the unresponsiveness to the chemotherapeutic drug pemetrexed (68), implying that miR-22 may be a telling serum predictor for monitoring the chemotherapeutic effects in NSCLC. Instead, the reduced expression of miR-22 frequently portend poor prognosis in patients with certain cancers. An example of this is that the downregulated miR-22 expression shows shorter overall survival time and is more likely to have a tendency of distant metastasis in cancer tissues than normal adjacent tissues in patients with hepatocellular carcinoma, gastric cancer and CRC $(46,69,70)$ (Table I).

Collectively, as for some cancers, monitoring the fluctuation of miR-22 expression in the serum or body fluids or the cancer tissues may be of great latent significance for cancer diagnosis, particularly early diagnosis, assessing therapeutic effects and prognosis, therefore, it is very promising that miR-22 may be eventually utilized as a predictive cancer biomarker for early accurate diagnosis, monitoring treatment responses in real-time and prognosis of outcomes and as an effective strategy for supplementary or even principal treatment in special cancers.

\section{Conclusions and prospects}

Numerous studies have revealed that miR-22 functions as either a tumor suppressor miRNA or an onco-miRNA to inhibit or promote tumor formation and malignant transformation from genetic to post-transcription level via intricate mechanisms, in which miR-22 could stimulate or turn off different cascades of events concerning pathways by directly or indirectly interacting with upstream or downstream molecules/pathways, either synergistically or antagonistically. Also, as the formation of miR-22-related positive or negative feedback loops extraordinarily amplify the inhibitory or promoting effects of miR-22 in a variety of cancers, therefore, miR-22 and countless related molecules constitute complex signaling networks where miR-22 is at the core of events (Table II). Indicating that miR-22 may serve as a hopeful therapeutic target for precision treatments in diverse cancers to inhibit proliferation, migration, invasion and metastasis, thus weakening or reversing chemoresistance to anticancer drugs. Besides, miR-22 expression in cancer cells and body fluids may fluctuate in different cancers and different growth stages in the same cancer, which makes it possible for miR-22 to be a potential and complementary or even independent biomarker in cancer diagnosis, monitoring treatment effects and prognosis.

However, there will be still many problems to be settled in the future due to the intricate and intrinsic mechanisms of miR-22 regulating cancer formation. For instance, the same cancer has different expression of miR-22 at the different growth steps, in which step does miR-22 play a primary or secondary role? Or inhibitory or promoting role or both? How does it interact with many other molecules? In particular, it is seemly paradoxical that miR-22 may show distinctively opposite effects (inhibition or promotion) on the biological behavior of different cancers by post-transcriptionally targeting the same transcription factors, such as PTEN. Therefore, further excavating the underlying mechanisms of miR-22 with many other molecules in manipulating tumor malignant progression may, to some degree, be very valuable for cancer diagnosis, treatment, and prognosis in precision medicine in the coming years. 
Table II. Molecular mechanisms of miR-22 regulating tumor progression.

\begin{tabular}{|c|c|c|c|c|c|}
\hline Tumor & miR-22 level & Target genes & Pathways & Effect & Refs. \\
\hline GC & $\downarrow$ & $\begin{array}{l}\text { MMP14 Snail } \\
\text { MTDH } \\
\text { CD151 Wnt-1 } \\
\text { SP1 }\end{array}$ & $\begin{array}{l}\text { EMT } \\
\text { Wnt/ } \beta \text {-catenin/ } \\
\text { TCF }\end{array}$ & - & $(19,20,36,41,57)$ \\
\hline $\mathrm{PC}$ & $\uparrow$ or $\downarrow$ & $\begin{array}{l}\text { PTEN Max } \\
\text { LAMC1 } \\
\text { ACLY }\end{array}$ & $\begin{array}{l}\text { PI3K/AKT } \\
\text { MAPK/ERK } \\
\text { PKC/ERK }\end{array}$ & + or - & $(7,9,11,16)$ \\
\hline $\mathrm{BC}$ & $\mathrm{N}$ or $\downarrow$ & $\begin{array}{l}\text { TIP60 GLUT1 } \\
\text { CD147 SIRT1 } \\
\text { CDK6 Sp1 } \\
\text { Erbb3 EVI-1 } \\
\text { ER } \alpha \text { MYCBP } \\
\text { PTEN Max } \\
\text { TET2 }\end{array}$ & $\begin{array}{l}\text { EMT } \\
\text { pRB pathway } \\
\text { PI3K/Akt } \\
\text { ER pathway } \\
\text { PKC/ERK }\end{array}$ & + or - & $(6,8,10,16,26,35,37,39,52)$ \\
\hline $\mathrm{RCC}$ & $\downarrow$ & SIRT1 PTEN & $\begin{array}{l}\text { Apoptosis } \\
\text { EMT }\end{array}$ & - & $(29,42)$ \\
\hline Liver cancer & $\downarrow$ & $\begin{array}{l}\text { Galectin-9 } \\
\text { CCNA2 } \\
\text { CDKN1A } \\
\text { ER } \alpha \text { HDAC4 } \\
\text { Zeb2 }\end{array}$ & $\begin{array}{l}\text { Apoptosis } \\
\text { Cell cycle }\end{array}$ & - & $(13,21,28,43)$ \\
\hline $\mathrm{CRC}$ & $\downarrow$ & $\begin{array}{l}\text { MDC1 } \\
\text { CCNA2 BTG1 } \\
\text { TIAM1 CDX2 } \\
\text { HIF-1 } \alpha \text { p21 } \\
\text { PTEN }\end{array}$ & $\begin{array}{l}\text { PI3K/AKT } \\
\text { Cell cycle } \\
\text { Autophagy } \\
\text { Apoptosis } \\
\text { Hypoxia }\end{array}$ & + or - & $(12,13,40,48,55,58,59,60)$ \\
\hline Lung cancer & $\downarrow$ or $\uparrow$ & $\begin{array}{l}\text { Erbb3 Max } \\
\text { ACLY }\end{array}$ & PKC/ERK & - & $(11,16,25)$ \\
\hline AML & $\downarrow$ & $\begin{array}{l}\text { CRTC1 } \\
\text { MYCBP }\end{array}$ & CREB/MYC & - & $(35,54)$ \\
\hline CLL & $\uparrow$ & PTEN & PI3K/AKT/FOXO1 & + & $(30)$ \\
\hline MDS & $\uparrow$ & TET2 & 5-hmc & + & $(53)$ \\
\hline CML & & NET1 & Cell cycle & - & $(22)$ \\
\hline Osteosarcoma & $\downarrow$ & $\begin{array}{l}\text { HMGB1 } \\
\text { ACLY }\end{array}$ & Autophagy & - & $(11,61)$ \\
\hline EEC & $\downarrow$ & $\mathrm{ER} \alpha$ & ER pathway & - & $(15)$ \\
\hline Medulloblastoma & $\downarrow$ & PAPST1 & $\begin{array}{l}\text { Cell proliferation } \\
\text { Apoptosis }\end{array}$ & - & $(23)$ \\
\hline Ewing sarcoma & $\downarrow$ & KDM3A & Histone demethylation & - & $(47)$ \\
\hline Cervical cancer & $\downarrow$ or $\uparrow$ & $\begin{array}{l}\text { TNFAIP8 } \\
\text { SIRT1 CDK6 } \\
\text { Sp1 PTEN } \\
\text { ACLY }\end{array}$ & $\begin{array}{l}\text { Apoptosis } \\
\text { pRB pathway } \\
\text { PI3K/AKT }\end{array}$ & + or - & $(6,8,11,45)$ \\
\hline Retinoblastoma & $\downarrow$ & Erbb3 & Cell proliferation & - & $(24)$ \\
\hline Ovarian cancer & $\downarrow$ & TIAM1 ESR1 EVI1 & PI3K/Akt/ mTOR & - & $(27,62)$ \\
\hline
\end{tabular}

GC, gastric cancer; PC, prostate cancer; BC, breast cancer; RCC, renal cell carcinoma; ESCC, esophageal squamous cell carcinoma; CLL, chronic lymphocytic leukemia; CRC, colorectal cancer; CML, chronic myeloid leukemia; EEC, endometrial endometrioid carcinoma; PC, prostate cancer; MDS, myelodysplastic syndrome; MMP14, member matrix metalloproteinase 14; MDC1, mediator of DNA damage checkpoint 1; CCNA2, cyclin A2; MTDH, metadherin; PTEN, phosphatase and tensin homolog; NET1, neuro-epithelial transforming gene 1; HMGB1, high-mobility group box 1; PAPST1, 3'-phosphoadenosine-5'-phosphosulfate transporter 1; KDM3A, histone K demethylase 3A; NSCLC, non-small cell lung cancer; CDKN1A, cyclin-dependent kinase inhibitor 1A; TIAM1, T-cell lymphoma invasion and metastasis 1; TNFAIP8, tumor necrosis factor- $\alpha$-induced protein 8; CDX2, caudal-type homeobox 2; Erbb3, erythoblastic leukemia viral oncogene homolog 3; EVI-1, ecotropic virus integration-1; MYCBP, c-Myc-binding protein; HDAC4, histone deacetylase 4; TET2, ten-eleven translocation 2; LAMC1, laminin $\gamma 1$; CRTC1, CREB-regulated transcription coactivator $1 ; \downarrow$, low level; $\uparrow$, high level; N, no change; +, promotion, -, inhibition. 


\section{Acknowledgements}

This study was supported by grants from the National Natural Science Foundation of China (81672730), the Zhejiang Provincial Natural Science Foundation (LY15H160067), the Jiaxing Municipal Science and Technology Project (2015AY23012, 2016AY23043) and Medical Key Discipline of Jiaxing (Pathology, 04-Z-01).

\section{References}

1. Lagos-Quintana M, Rauhut R, Lendeckel W and Tuschl T: Identification of novel genes coding for small expressed RNAs. Science 294: 853-858, 2001.

2. Knyazev EN, Samatov TR, Fomicheva KA, Nyushko KM, Alekseev BY and Shkurnikov MY: MicroRNA hsa-miR-4674 in hemolysis-free blood plasma is associated with distant metastases of prostatic cancer. Bull Exp Biol Med 161: 112-115, 2016.

3. Yang C, Ning S, Li Z, Qin X and Xu W: miR-22 is down-regulated in esophageal squamous cell carcinoma and inhibits cell migration and invasion. Cancer Cell Int 14: 138, 2014.

4. Damavandi Z, Torkashvand S, Vasei M, Soltani BM, Tavallaei M and Mowla SJ: Aberrant expression of breast developmentrelated microRNAs, miR-22, miR-132, and miR-212, in breast tumor tissues. J Breast Cancer 19: 148-155, 2016.

5. Yang M, Jiang N, Cao QW and Sun Q: EDD1 predicts prognosis and regulates gastric cancer growth in vitro and in vivo via miR-22. Biol Chem: Apr 28, 2016 (Epub ahead of print). doi: $10.1515 / \mathrm{hsz}-2015-0279$.

6. Bar N and Dikstein R: miR-22 forms a regulatory loop in PTEN/ AKT pathway and modulates signaling kinetics. PLoS One 5: e10859, 2010.

7. Budd WT, Seashols-Williams SJ, Clark GC, Weaver D, Calvert V, Petricoin E, Dragoescu EA, O'Hanlon K and Zehner ZE: Dual action of miR-125b as a tumor suppressor and oncomiR-22 promotes prostate cancer tumorigenesis. PLoS One 10: e0142373, 2015.

8. Xu D, Takeshita F, Hino Y, Fukunaga S, Kudo Y, Tamaki A, Matsunaga J, Takahashi RU, Takata T, Shimamoto A, et al: miR-22 represses cancer progression by inducing cellular senescence. J Cell Biol 193: 409-424, 2011

9. Pasqualini L, Bu H, Puhr M, Narisu N, Rainer J, Schlick B, Schäfer G, Angelova M, Trajanoski Z, Börno ST, et al: miR-22 and miR-29a are members of the androgen receptor cistrome modulating LAMC1 and $\mathrm{Mcl}-1$ in prostate cancer. Mol Endocrinol 29: 1037-1054, 2015.

10. Chen B, Tang H, Liu X, Liu P, Yang L, Xie X, Ye F, Song C, $\mathrm{Xie} \mathrm{X}$ and Wei W: miR-22 as a prognostic factor targets glucose transporter protein type 1 in breast cancer. Cancer Lett 356 410-417, 2015.

11. Xin M, Qiao Z, Li J, Liu J, Song S, Zhao X, Miao P, Tang T, Wang L, Liu W, et al: miR-22 inhibits tumor growth and metastasis by targeting ATP citrate lyase: Evidence in osteosarcoma prostate cancer, cervical cancer and lung cancer. Oncotarget 7: 44252-44265, 2016.

12. Yamakuchi M, Yagi S, Ito T and Lowenstein CJ: MicroRNA-22 regulates hypoxia signaling in colon cancer cells. PLoS One 6: e20291, 2011.

13. Yang F, Hu Y, Liu HX and Wan YJ: miR-22-silenced cyclin A expression in colon and liver cancer cells is regulated by bile acid receptor. J Biol Chem 290: 6507-6515, 2015.

14. Shi $C$ and $X u$ X: MicroRNA-22 is down-regulated in hepatitis $B$ virus-related hepatocellular carcinoma. Biomed Pharmacother 67: 375-380, 2013.

15. Li S, Hu R, Wang C, Guo F, Li X and Wang S: miR-22 inhibits proliferation and invasion in estrogen receptor $\alpha$-positive endometrial endometrioid carcinomas cells. Mol Med Rep 9: 2393-2399, 2014

16. Ting Y, Medina DJ, Strair RK and Schaar DG: Differentiationassociated miR-22 represses Max expression and inhibits cell cycle progression. Biochem Biophys Res Commun 394: 606-611, 2010.

17. Alvarez-Díaz S, Valle N, Ferrer-Mayorga G, Lombardía L, Herrera M, Domínguez O, Segura MF, Bonilla F, Hernando E and Muñoz A: MicroRNA-22 is induced by vitamin D and contributes to its antiproliferative, antimigratory and gene regulatory effects in colon cancer cells. Hum Mol Genet 21: 2157-2165, 2012.
18. Pandey DP and Picard D: miR-22 inhibits estrogen signaling by directly targeting the estrogen receptor alpha mRNA. Mol Cell Biol 29: 3783-3790, 2009.

19. Wang X, Yu H, Lu X, Zhang P, Wang M and Hu Y: miR-22 suppresses the proliferation and invasion of gastric cancer cells by inhibiting CD151. Biochem Biophys Res Commun 445: 175-179, 2014.

20. Tang Y, Liu X, Su B, Zhang Z, Zeng X, Lei Y, Shan J, Wu Y, Tang $\mathrm{H}$ and $\mathrm{Su} \mathrm{Q}$ : microRNA-22 acts as a metastasis suppressor by targeting metadherin in gastric cancer. Mol Med Rep 11: 454-460, 2015.

21. Yang Q, Jiang W, Zhuang C, Geng Z, Hou C, Huang D, Hu L and Wang X: microRNA-22 downregulation of galectin-9 influences lymphocyte apoptosis and tumor cell proliferation in liver cancer. Oncol Rep 34: 1771-1778, 2015.

22. Ahmad HM, Muiwo P, Ramachandran SS, Pandey P, Gupta YK, Kumar L, Kulshreshtha R and Bhattacharya A: miR-22 regulates expression of oncogenic neuro-epithelial transforming gene 1 , NET1. FEBS J 281: 3904-3919, 2014.

23. Xu QF, Pan YW, Li LC, Zhou Z, Huang QL, Pang JC, Zhu XP, Ren Y, Yang H, Ohgaki H, et al: miR-22 is frequently downregulated in medulloblastomas and inhibits cell proliferation via the novel target PAPST1. Brain Pathol 24: 568-583, 2014.

24. Sreenivasan S, Thirumalai K, Danda R and Krishnakumar S: Effect of curcumin on miRNA expression in human Y79 retinoblastoma cells. Curr Eye Res 37: 421-428, 2012.

25. Ling B, Wang GX, Long G, Qiu JH and Hu ZL: Tumor suppressor miR-22 suppresses lung cancer cell progression through posttranscriptional regulation of ErbB3. J Cancer Res Clin Oncol 138: 1355-1361, 2012.

26. Patel JB, Appaiah HN, Burnett RM, Bhat-Nakshatri P, Wang G, Mehta R, Badve S, Thomson MJ, Hammond S, Steeg P, et al: Control of EVI-1 oncogene expression in metastatic breast cancer cells through microRNA miR-22. Oncogene 30: 1290-1301, 2011.

27. Li J, Liang S, Yu H, Zhang J, Ma D and Lu X: An inhibitory effect of miR-22 on cell migration and invasion in ovarian cancer. Gynecol Oncol 119: 543-548, 2010.

28. Jiang R, Deng L, Zhao L, Li X, Zhang F, Xia Y, Gao Y, Wang $X$ and Sun B: miR-22 promotes HBV-related hepatocellular carcinoma development in males. Clin Cancer Res 17: 5593-5603, 2011.

29. Fan W, Huang J, Xiao H and Liang Z: MicroRNA-22 is downregulated in clear cell renal cell carcinoma, and inhibits cell growth, migration and invasion by targeting PTEN. Mol Med Rep 13: 4800-4806, 2016.

30. Palacios F, Abreu C, Prieto D, Morande P, Ruiz S, FernándezCalero T, Naya H, Libisch G, Robello C, Landoni AI, et al: Activation of the PI3K/AKT pathway by microRNA-22 results in CLL B-cell proliferation. Leukemia 29: 115-125, 2015.

31. Tang J, Li Y, Wang J, Wen Z, Lai M and Zhang H: Molecular mechanisms of microRNAs in regulating epithelial-mesenchymal transitions in human cancers. Cancer Lett 371: 301-313, 2016.

32. Lu M, Jolly MK, Levine H, Onuchic JN and Ben-Jacob E: MicroRNA-based regulation of epithelial-hybrid-mesenchymal fate determination. Proc Natl Acad Sci USA 110: 18144-18149, 2013.

33. Moes M, Le Béchec A, Crespo I, Laurini C, Halavatyi A, Vetter G, Del Sol A and Friederich E: A novel network integrating a miRNA-203/SNAI1 feedback loop which regulates epithelial to mesenchymal transition. PLoS One 7: e35440, 2012.

34. Ding X, Park SI, McCauley LK and Wang CY: Signaling between transforming growth factor $\beta$ (TGF- $\beta$ ) and transcription factor SNAI2 represses expression of microRNA miR-203 to promote epithelial-mesenchymal transition and tumor metastasis. J Biol Chem 288: 10241-10253, 2013.

35. Xiong J, Du Q and Liang Z: Tumor-suppressive microRNA-22 inhibits the transcription of E-box-containing c-Myc target genes by silencing c-Myc binding protein. Oncogene 29: 4980-4988, 2010.

36. Guo MM, Hu LH, Wang YQ, Chen P, Huang JG, Lu N, He JH and Liao CG: miR-22 is down-regulated in gastric cancer, and its overexpression inhibits cell migration and invasion via targeting transcription factor Sp1. Med Oncol 30: 542, 2013.

37. Kong LM, Liao CG, Zhang Y, Xu J, Li Y, Huang W, Zhang Y, Bian $\mathrm{H}$ and Chen $\mathrm{ZN}$ : A regulatory loop involving miR-22, Sp1, and c-Myc modulates CD147 expression in breast cancer invasion and metastasis. Cancer Res 74: 3764-3778, 2014. 
38. Choi JH, Hwang YP, Kim HG, Khanal T, Do MT, Jin SW, Han HJ, Lee HS, Lee YC, Chung YC, et al: Saponins from the roots of Platycodon grandiflorum suppresses TGF $\beta 1$-induced epithelial-mesenchymal transition via repression of PI3K/Akt, ERK1/2 and Smad2/3 pathway in human lung carcinoma A549 cells. Nutr Cancer 66: 140-151, 2014.

39. Pandey AK, Zhang Y, Zhang S, Li Y, Tucker-Kellogg G, Yang H and Jha S: TIP60-miR-22 axis as a prognostic marker of breast cancer progression. Oncotarget 6: 41290-41306, 2015.

40. Li B, Song Y, Liu TJ, Cui YB, Jiang Y, Xie ZS and Xie SL: miRNA-22 suppresses colon cancer cell migration and invasion by inhibiting the expression of T-cell lymphoma invasion and metastasis 1 and matrix metalloproteinases 2 and 9. Oncol Rep 29: 1932-1938, 2013

41. Zuo QF, Cao LY, Yu T, Gong L, Wang LN, Zhao YL, Xiao B and Zou QM: MicroRNA-22 inhibits tumor growth and metastasis in gastric cancer by directly targeting MMP14 and Snail. Cell Death Dis 6: e2000, 2015.

42. Zhang S, Zhang D, Yi C, Wang Y, Wang H and Wang J: MicroRNA-22 functions as a tumor suppressor by targeting SIRT1 in renal cell carcinoma. Oncol Rep 35: 559-567, 2016.

43. Su YH, Huang WC, Huang TH, Huang YJ, Sue YK, Huynh TT, Hsiao M, Liu TZ, Wu AT and Lin CM: Folate deficient tumor microenvironment promotes epithelial-to-mesenchymal transition and cancer stem-like phenotypes. Oncotarget 7: 33246-33256, 2016.

44. Ninomiya S, Tyybäkinoja A, Borze I, Räty R, SaarinenPihkala UM, Usvasalo A, Elonen E and Knuutila S: Integrated analysis of gene copy number, copy neutral $\mathrm{LOH}$, and microRNA profiles in adult acute lymphoblastic leukemia. Cytogenet Genome Res 136: 246-255, 2012.

45. Shi TY, Cheng X, Yu KD, Sun MH, Shao ZM, Wang MY, Zhu ML, He J, Li QX, Chen XJ, et al: Functional variants in TNFAIP8 associated with cervical cancer susceptibility and clinical outcomes. Carcinogenesis 34: 770-778, 2013.

46. Zhang J, Yang Y, Yang T, Liu Y, Li A, Fu S, Wu M, Pan Z and Zhou W: microRNA-22, downregulated in hepatocellular carcinoma and correlated with prognosis, suppresses cell proliferation and tumourigenicity. Br J Cancer 103: 1215-1220, 2010.

47. Parrish JK, Sechler M, Winn RA and Jedlicka P: The histone demethylase KDM3A is a microRNA-22-regulated tumor promoter in Ewing Sarcoma. Oncogene 34: 257-262, 2015.

48. Lee JH, Park SJ, Jeong SY, Kim MJ, Jun S, Lee HS, Chang IY, Lim SC, Yoon SP, Yong J, et al: MicroRNA-22 suppresses DNA repair and promotes genomic instability through targeting of MDC1. Cancer Res 75: 1298-1310, 2015.

49. Madzo J, Liu H, Rodriguez A, Vasanthakumar A, Sundaravel S, Caces DB, Looney TJ, Zhang L, Lepore JB, Macrae T, et al: Hydroxymethylation at gene regulatory regions directs stem/ early progenitor cell commitment during erythropoiesis. Cell Rep 6: 231-244, 2014.

50. Shen L, Wu H, Diep D, Yamaguchi S, D'Alessio AC, Fung HL, Zhang $\mathrm{K}$ and Zhang Y: Genome-wide analysis reveals TET- and TDG-dependent 5-methylcytosine oxidation dynamics. Cell 153 692-706, 2013

51. Coutinho DF, Monte-Mór BC, Vianna DT, Rouxinol ST, Batalha AB, Bueno AP, Boulhosa AM, Fernandez TS, Pombode-Oliveira MS, Gutiyama LM, et al: TET2 expression level and 5 -hydroxymethylcytosine are decreased in refractory cytopenia of childhood. Leuk Res 39: 1103-1108, 2015.

52. Song SJ, Poliseno L, Song MS, Ala U, Webster K, Ng C, Beringer G, Brikbak NJ, Yuan X, Cantley LC, et al: MicroRNAantagonism regulates breast cancer stemness and metastasis via TET-family-dependent chromatin remodeling. Cell 154: 311-324, 2013.

53. Song SJ, Ito K, Ala U, Kats L, Webster K, Sun SM, JongenLavrencic M, Manova-Todorova K, Teruya-Feldstein J, Avigan DE, et al: The oncogenic microRNA miR-22 targets the TET2 tumor suppressor to promote hematopoietic stem cell selfrenewal and transformation. Cell Stem Cell 13: 87-101, 2013.

54. Jiang X, Hu C, Arnovitz S, Bugno J, Yu M, Zuo Z, Chen P, Huang H, Ulrich B, Gurbuxani S, et al: miR-22 has a potent antitumour role with therapeutic potential in acute myeloid leukemia. Nat Commun 7: 11452, 2016
55. Tagawa T, Haraguchi T, Hiramatsu H, Kobayashi K, Sakurai K, Inada $\mathrm{K}$ and Iba $\mathrm{H}$ : Multiple microRNAs induced by $\mathrm{Cdx} 1$ suppress $\mathrm{Cdx} 2$ in human colorectal tumour cells. Biochem J 447: 449-455, 2012

56. Marzi MJ, Puggioni EM, Dall'Olio V, Bucci G, Bernard L, Bianchi F, Crescenzi M, Di Fiore PP and Nicassio F: Differentiation-associated microRNAs antagonize the Rb-E2F pathway to restrict proliferation. J Cell Biol 199: 77-95, 2012.

57. Tang H, Kong Y, Guo J, Tang Y, Xie X, Yang L, Su Q and Xie X: Diallyl disulfide suppresses proliferation and induces apoptosis in human gastric cancer through Wnt-1 signaling pathway by up-regulation of miR-200b and miR-22. Cancer Lett 340: 72-81, 2013.

58. Li J, Zhang Y, Zhao J, Kong F and Chen Y: Overexpression of miR-22 reverses paclitaxel-induced chemoresistance through activation of PTEN signaling in p53-mutated colon cancer cells. Mol Cell Biochem 357: 31-38, 2011.

59. Tsuchiya N, Izumiya $M$, Ogata-Kawata $H$, Okamoto $K$, Fujiwara Y, Nakai M, Okabe A, Schetter AJ, Bowman ED, Midorikawa Y, et al: Tumor suppressor miR-22 determines p53-dependent cellular fate through post-transcriptional regulation of p21. Cancer Res 71: 4628-4639, 2011.

60. Zhang H, Tang J, Li C, Kong J, Wang J, Wu Y, Xu E and Lai M: miR-22 regulates 5-FU sensitivity by inhibiting autophagy and promoting apoptosis in colorectal cancer cells. Cancer Lett 356 781-790, 2015

61. Guo S, Bai R, Liu W, Zhao A, Zhao Z, Wang Y, Wang Y, Zhao W and Wang W: miR-22 inhibits osteosarcoma cell proliferation and migration by targeting HMGB1 and inhibiting HMGB1mediated autophagy. Tumour Biol 35: 7025-7034, 2014.

62. Nagaraja AK, Creighton CJ, Yu Z, Zhu H, Gunaratne PH, Reid JG, Olokpa E, Itamochi H, Ueno NT, Hawkins SM, et al: A link between miR-100 and FRAP1/mTOR in clear cell ovarian cancer. Mol Endocrinol 24: 447-463, 2010.

63. Kawahigashi Y, Mishima T, Mizuguchi Y, Arima Y, Yokomuro S, Kanda T, Ishibashi O, Yoshida H, Tajiri T and Takizawa T: MicroRNA profiling of human intrahepatic cholangiocarcinoma cell lines reveals biliary epithelial cell-specific microRNAs. J Nippon Med Sch 76: 188-197, 2009.

64. Zekri AN, Youssef AS, El-Desouky ED, Ahmed OS, Lotfy MM, Nassar AA and Bahnassey AA: Serum microRNA panels as potential biomarkers for early detection of hepatocellular carcinoma on top of HCV infection. Tumour Biol 37: 12273-12286, 2016.

65. Shin YM, Yun J, Lee OJ, Han HS, Lim SN, An JY, Lee KH, Lee KM and Choe KH: Diagnostic value of circulating extracellular miR-134, miR-185, and miR-22 levels in lung adenocarcinoma-associated malignant pleural effusion. Cancer Res Treat 46: 178-185, 2014

66. Zhang C, Wang C, Chen X, Yang C, Li K, Wang J, Dai J, Hu Z, Zhou X, Chen L, et al: Expression profile of microRNAs in serum: A fingerprint for esophageal squamous cell carcinoma. Clin Chem 56: 1871-1879, 2010.

67. Ganepola GA, Rutledge JR, Suman P, Yiengpruksawan A and Chang DH: Novel blood-based microRNA biomarker panel for early diagnosis of pancreatic cancer. World J Gastrointest Oncol 6: 22-33, 2014.

68. Franchina T, Amodeo V, Bronte G, Savio G, Ricciardi GR, Picciotto M, Russo A, Giordano A and Adamo V: Circulating miR-22, miR-24 and miR-34a as novel predictive biomarkers to pemetrexed-based chemotherapy in advanced non-small cell lung cancer. J Cell Physiol 229: 97-99, 2014.

69. Wang W, Li F, Zhang Y, Tu Y, Yang Q and Gao X: Reduced expression of miR-22 in gastric cancer is related to clinicopathologic characteristics or patient prognosis. Diagn Pathol 8: 102, 2013.

70. Zhang G, Xia S, Tian H, Liu Z and Zhou T: Clinical significance of miR-22 expression in patients with colorectal cancer. Med Oncol 29: 3108-3112, 2012. 